Review

\title{
Novel insights into cardiomyocytes provided by atomic force microscopy
}

\author{
Daniele Borin $^{\mathrm{a}, 1}$, Ilaria Pecorari ${ }^{\mathrm{a}, 1}$, Brisa Pena ${ }^{\mathrm{b}, \mathrm{c}}$, Orfeo Sbaizero $^{\mathrm{a}, *}$ \\ a Department of Engineering and Architecture, University of Trieste, Via Valerio 10, 34127, Trieste, Italy \\ ${ }^{b}$ Cardiovascular Institute, University of Colorado Denver, E 17th Pl, Aurora, CO 80045, United States \\ c Bioengineering Department, University of Colorado Denver, E 17th Pl, Aurora, CO 80045, United States
}

\section{A R T I C L E I N F O}

\section{Article history:}

Received 30 May 2017

Received in revised form 29 June 2017

Accepted 3 July 2017

Available online 4 July 2017

\section{Keywords:}

Atomic force microscopy

Cardiomyocyte

Biomechanical properties

Mechanotransduction

Beating activity

\begin{abstract}
A B S T R A C T
Cardiovascular diseases (CVDs) are the number one cause of death globally, therefore interest in studying aetiology, hallmarks, progress and therapies for these disorders is constantly growing. Over the last decades, the introduction and development of atomic force microscopy (AFM) technique allowed the study of biological samples at the micro- and nanoscopic level, hence revealing noteworthy details and paving the way for investigations on physiological and pathological conditions at cellular scale.

The present work is aimed to collect and review the literature on cardiomyocytes (CMs) studied by AFM, in order to emphasise the numerous potentialities of this approach and provide a platform for researchers in the field of cardiovascular diseases. Original data are also presented to highlight the application of AFM to characterise the viscoelastic properties of CMs.
\end{abstract}

(c) 2017 Elsevier Ltd. All rights reserved.

\section{Contents}

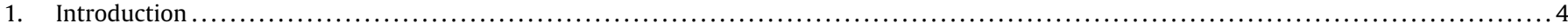

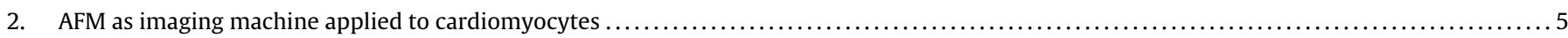

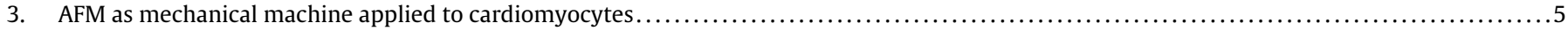

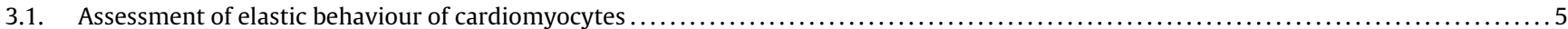

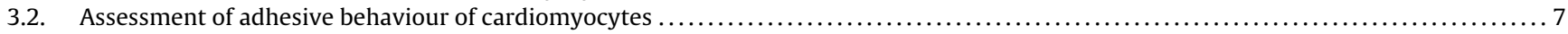

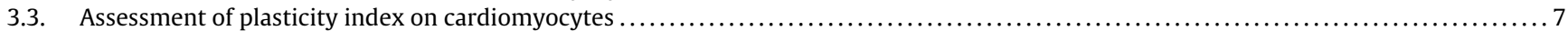

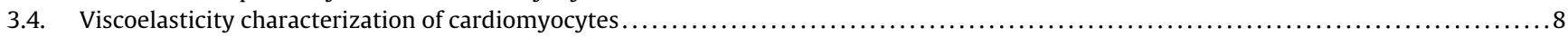

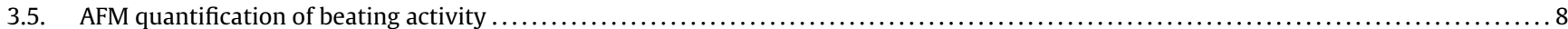

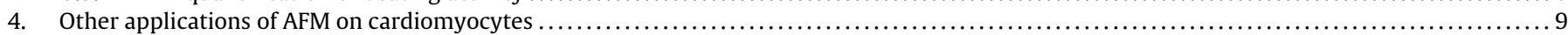

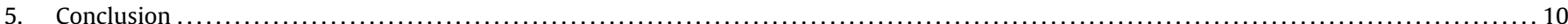

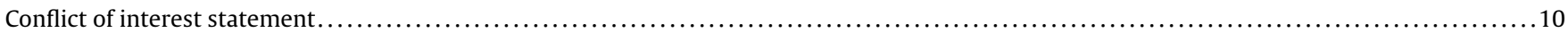

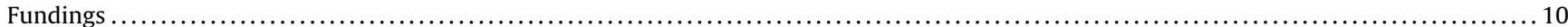

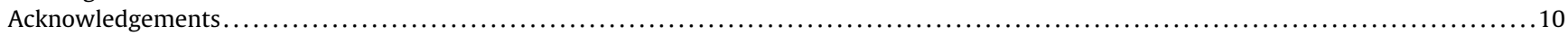

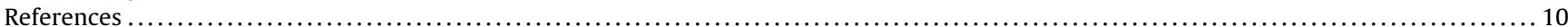

\section{Introduction}

In the late 1980s, the atomic force microscope (AFM) was developed by Binnig et al. [1] to investigate surfaces of insulators at the atomic scale, while avoiding damages to the specimens. AFM

\footnotetext{
* Corresponding author at: Department of Engineering and Architecture University of Trieste, Piazzale Europa 1, 34127 Trieste, Italy.

E-mail address: sbaizero@units.it(O. Sbaizero).

1 Share co-authorship.
}

is a scanning probe technique, which exploits the deflections of a cantilevered spring to image and probe different kind of samples. Fundamental components and working principles of the AFM are shown in Fig. 1.

The atomic force microscope has proven to be a versatile tool of investigation, since it can either acquire a sample topography or measure various mechanical properties, such as stiffness, adhesive and viscoelastic behaviour; in the former case, the AFM works as an imaging machine, whereas in the latter it can be considered a mechanical instrument [2]. In addition, as the AFM can work in aqueous environments, it was forthwith thought to be used for 


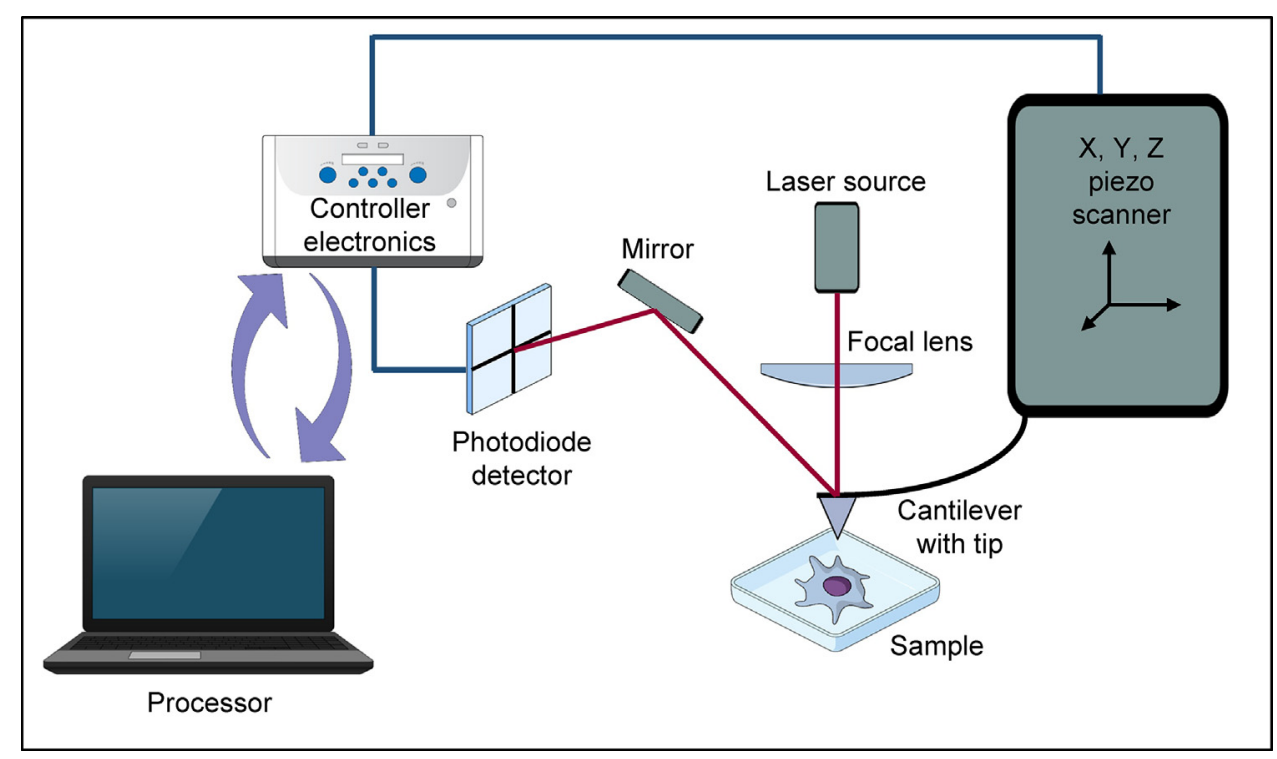

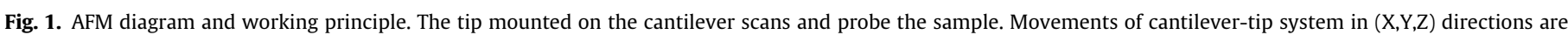

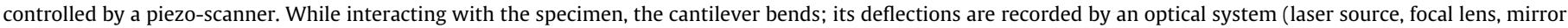
and photodiode detector). The controller electronics provides a feedback loop that permits to adjust the tip-sample separation.

studying biological specimens either close to or under physiological conditions.

Cardiovascular diseases are still the leading cause of death worldwide. Particularly, according to Gillespie et al. [3], heart disease and stroke are the first and fourth causes of death, respectively, in the United States. The proper functioning of the heart is pivotal for living organisms, and cardiology research is mainly focused on the thorough comprehension of bio-chemo-mechanical principles underlying the physiological behaviour of the heart. Yet, the effects of inherited or incidental cardiac dysfunctions are under investigation, likewise any possible therapy, such as drug administration and tissue regeneration/transplantation. The study of aetiology, hallmarks and progress of a cardiac pathology can be considered a multidisciplinary task, since it involves physicians, biologists, and more recently biophysicists and bioengineers. Altogether, they investigate cardiac behaviour both at the macroscopic, e.g. via echocardiography or ventriculography, and microscopic level.

In recent years, more and more researchers focus on the basic constituent of cardiac tissue, mainly on cardiomyocytes (CMs), which are the core unit of the heart, generating and transmitting the contractile force. In this scenario, AFM can play a key role, due to its high resolution, both in topographic and force terms. The present work aims to review the studies that involved AFM technology to investigate the structure and function of CMs. In addition, original data are presented as further characterization of cardiomyocytes in terms of plasticity index and stress relaxation behaviour.

\section{AFM as imaging machine applied to cardiomyocytes}

Over the years, various biological samples have been characterized for their topography by AFM, as thoroughly reviewed in [4,5]. Regarding the aim of the present work, in 1999, Perez-Terzic et al. [6] imaged sarcolemma-stripped CMs from neonatal rats, focusing on the nuclear pore complexes (NPCs). Data showed that both $\mathrm{Ca}^{2+}$ and ATP/GTP depletion induced a significant decrease in the percentage of open NPCs compared to untreated CMs, the second treatment resulting also in more relaxed pores (bigger outer diameter).

In 2001, Davis et al. [7] resolved both external and sub-surface features of fixed, intact cardiomyocytes from the ventricles of adult guinea pigs, thus paving the way for further AFM investigations of whole cells. More recently, the nanoscopic resolution of this technique allowed to detect changes in the plasma membrane surface of neonatal murine CMs induced by administration of aldosterone [8]. Similarly, neonatal rat CMs were treated with GCIP-27, a synthetic polypeptide thought to prevent cardiac hypertrophy, and exhibited an increased surface roughness compared to the not-treated, control group [9]. Another study analysed the cell surface and confirmed the absence of t-tubules in mouse and human embryonic stem cell-derived cardiomyocytes (ESC-CMs), which were compared with adult guinea pig ventricular CMs [10]. Moreover, AFM revealed the cell surface properties of cardiomyocytes from healthy and failing mice hearts: the former were characterized by a periodic succession of crests and hollows, while the latter showed a general loss of these structures [11].

In a ground-breaking study, AFM was applied to observe the cytoskeleton of murine CMs stimulated by lipopolysaccharide (LPS), which is known to be related to sepsis and, therefore, to cardiac dysfunction. The acquired images highlighted the cytoskeleton reorganization and the morphological changes induced by LPS, thus proving the use of AFM to study the functional properties through the observation of structural information [12].

The atomic force microscopy was then exploited to investigate infarcted porcine myocardium treated with mesenchymal stem cells (MSCs), either with or without glucagon-like peptide1 (GLP-1) expression [13]. Interestingly, Wright et al. [13] found that collagen fibril diameter significantly increased after treatment with MSCs, further demonstrating that the MSCs therapy option can have a dramatic effect on the scar deposited as a consequence of myocardial infarction.

\section{AFM as mechanical machine applied to cardiomyocytes}

\subsection{Assessment of elastic behaviour of cardiomyocytes}

Early after its invention, the atomic force microscope was used to assess the mechanical properties, namely hardness, elastic and plastic behaviour, by nano-indentation of the investigated sample [14]. The mechanical analysis by AFM at the atomic level has raised great interest in the field of mechanotransduction, which is the 
Dwell time $=0$

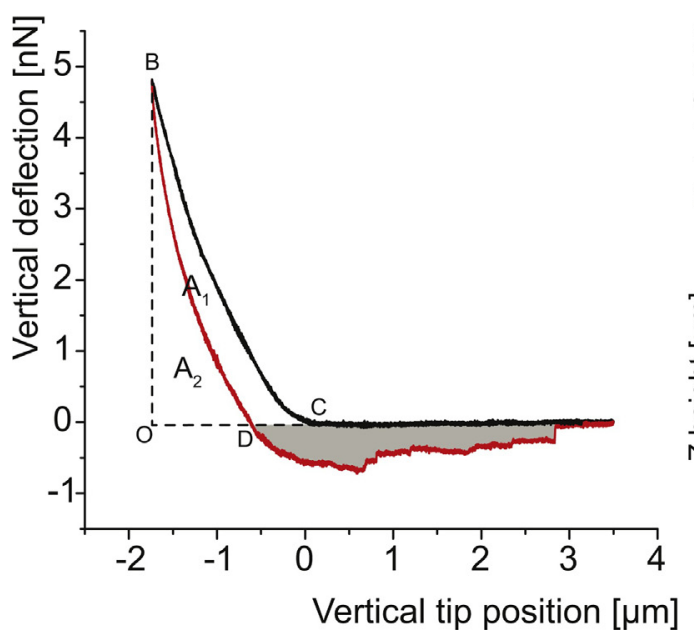

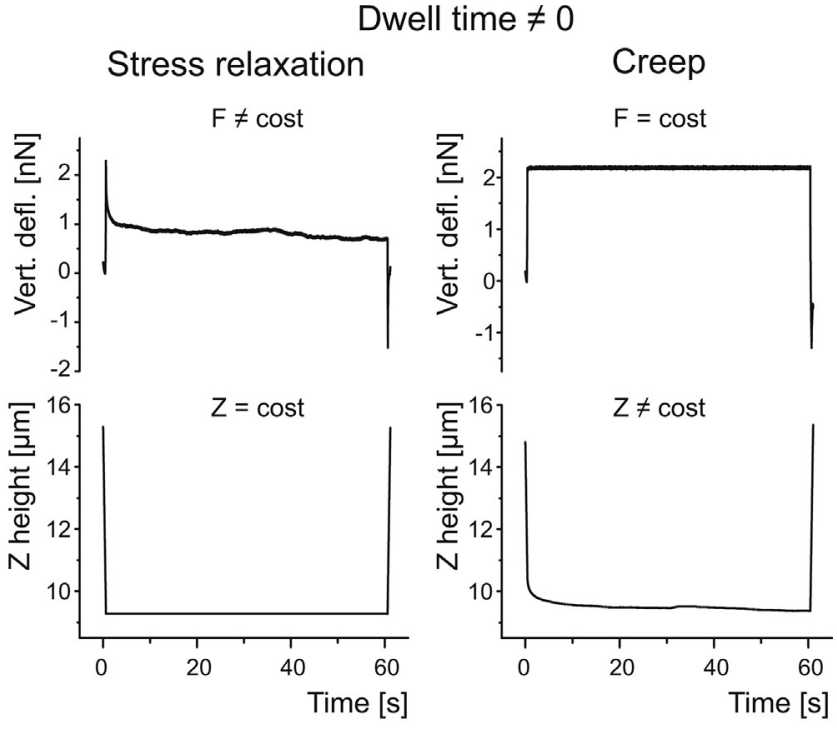

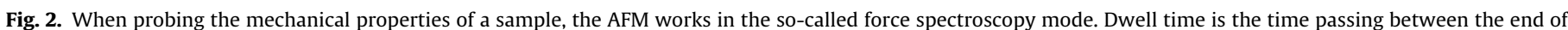

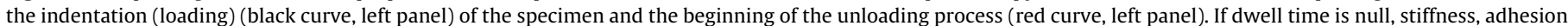

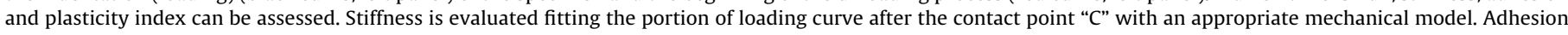

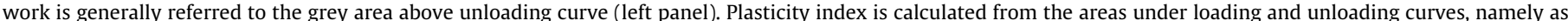

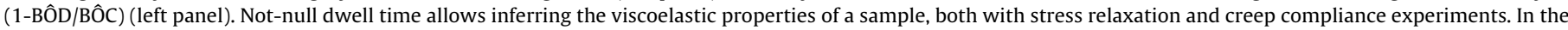

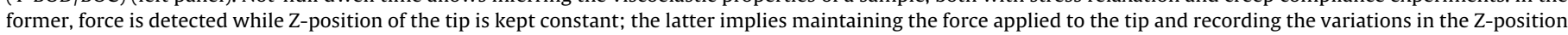
of the probe (right panel).

study of cellular and molecular processes that convert mechanical cues into biochemical signals [15].

A large number of studies on the elastic properties of living cells, commonly described as Young's modulus, has been issued. Young's modulus is a measure of the material's stiffness, that is the aptitude of a sample to be deformed. Basically, the AFM can act as an indenter, thus can perform force measurements on a desired portion of a cell while detecting how the specimen deforms in response to the applied force (see Fig. 2). Nevertheless, the estimation of cell stiffness is always affected by several factors, for instance the choice of the most suitable mechanical model to fit the experimental data, the assumptions on which that model relies, and the precision in determining the contact point (i.e. the point in the force curve at which the AFM probe reaches the specimen). A thorough discussion about this topic is provided in [16].

Adult rat atrial myocytes were nano-mechanically characterized by AFM both in their quiescent and contractile status, along their overall surface (i.e. nuclear and peripheral regions), and under different conditions (i.e. either with different extracellular calcium concentrations or after fixation) [17]. Also adult male mouse CMs were tested in their relaxed and contractile state, exploiting either high- $\mathrm{K}^{+}$solution or low- $\mathrm{Ca}^{2+}$ solution with EGTA and 2,3-butanedione monoxime [18]. Spontaneously beating, neonatal rat cardiomyocytes were studied with AFM in their systole and diastole by Azeloglu and Costa [19], revealing the spatial heterogeneity of mechanical properties within a single cell and the increase of CMs stiffness during contraction than relaxation. The same study demonstrated that administration of blebbistain, a specific inhibitor of actin-myosin interaction in cardiac muscle, reduced the elastic modulus of CMs to diastolic values; the authors also outlined that increasing the culture time of cells resulted in the stiffening of both systolic and diastolic moduli, although in the last case, the variation was not statistically significant. Saenz-Cogollo and colleagues [20] grew a confluent layer of rat embryos CMs on microelectrode arrays (MEA) and coupled the system with AFM. Such setup allowed the recording of extracellular action potential signal and the synchronization of the contractile cycle with mor- phological and mechanical measurements. Similarly to what was reported in [19], a substantial stiffening of cells during contraction was observed, followed by softening in the relaxation phase.

Dague et al. [11] studied the effects induced by formamide, which is known to cause detubulation, both on topography and elastic properties of murine cardiomyocytes. They found that the response of cells to various concentrations of formamide mimics the phenotype of CMs from failed hearts at different stages. In another study, CMs derived from chicken embryos were analysed after dosing cytochalasin B, in order to determine the influence of the actin network on the Young's modulus of these cells [21]. Here, a consistent decrease of the elastic modulus was observed after actin depolymerization.

Anticancer drugs are well-known to cause cardiotoxicity, ergo primary adult murine CMs were exposed to an anthracycline anticancer drug (doxorubicin) and a cardioprotective agent (dexrazoxane), and cell stiffness was assessed to elucidate the effects of these two drugs at the cellular level [22]. Doxorubicin treated CMs displayed the same stiffness of control CMs within the first $50 \mathrm{~min}$, followed by a significant softening, whereas pre-treatment with dexrazoxane avoided these variations.

Besides the outcome of various chemical substances in the mechanical properties of CMs, the effect of a gradient of substrate rigidity in the elasticity of neonatal rat ventricular myocytes (NRVMs) was investigated [23], resulting in a non-significant influence.

The AFM was also exploited to study the role of different cytoskeletal proteins in maintaining the structure and function of cells, as in the case of vinculin. Cardiomyocytes from vinculin heterozygous null mice were softer than cells from their wild type littermate; yet, rescue of vinculin restored the elastic modulus to control levels, as demonstrated by Tangney et al. [24]. Adult CMs from ephrin-B1 knock-out mice were found to be stiffer than their counterpart derived from wild type animals, as resulted from the destabilization of the lateral membrane induced by the absence of the considered membrane protein [25]. 
Tangney et al. [24] investigated vinculin because its mutations are related to hypertrophic and dilated cardiomyopathy (DCM), thus suggesting the AFM as a valuable tool to investigate pathological conditions and the effects of drug therapies. Several other studies support this application: for instance, ageing was related to left ventricular diastolic dysfunction and was demonstrated to be crucial for the stiffness of rat CMs; values for 30-months-old male animals were higher than those from 4-months-old rats [26]. The increase of cell stiffness with age was confirmed also in intact, enzymatically isolated cardiomyocytes derived from aged and young murine hearts under $\mathrm{Ca}^{2+}$-free conditions [27]. Similarly, diabetes induced a stiffening in adult mice CMs, as observed in [28]. In addition, the Young's modulus of NRVMs was assessed to elucidate the effects of genetic mutations in A-type lamin, which are responsible for a particular class of pathologies called laminopathies. Lanzicher et al. [29] showed that NRVMs infected with human D192G LMNA were stiffer than wild type (expressing wild type human lamin) and control (not-treated) counterparts. Moreover, cell stiffness was investigated in hypertrophied hearts: adult male rats were treated with either a vehicle (control) or isoproterenol (ISO), resulting in an increase of the elastic modulus of the ISO group compared to the control one [30]. Another cardiopathological condition,the heart failure, was studied from a mechanical point of view, exploiting the unique properties of the AFM. Indeed, Dague et al. [11] compared the elasticity of cardiomyocytes from healthy and failing mice hearts, finding that early after heart failure, CMs decreased their Young's modulus, whereas at later stages, cells became stiffer than controls. Likewise, Liu et al. [31] exploited the AFM to compare the properties of induced pluripotent stem cells derived cardiomyocytes (iPSC-CM) from healthy subjects and patients affected by dilated cardiomyopathy. The authors demonstrated that DCM led to a decrease in iPSC-CMs elasticity. It is noteworthy to stress that in the last study, cardiomyocytes were derived from human skin biopsies, whereas most of the reported works used animal models. Growing efforts have been recently made to develop protocols for obtaining CMs, e.g. iPSC-CM, that resembles those found in native human heart tissue. Indeed, this approach could overcome the limitations imposed by the differences between human and animal models and the obvious challenges faced to obtain $\mathrm{CMs}$ from heart biopsies.

It is also noteworthy to mention that the deformability of cardiomyocytes was assessed even under nonconventional conditions, like after antiorthostatic suspension $[32,33]$ or 2-g centrifugation [34].

Some of the reviewed works applied the Hertz model or its variants to quantify the Young's modulus of cardiac cells $[11,23,24,29,30,35]$. Nevertheless, the suitability of these models for biological specimens has been questioned. A better description of cellular stiffness was proposed by Soufivand and colleagues [36], using hyperelastic models to fit data from embryonic murine CMs. Furthermore, nonlinear elastic behaviour of rabbit CMs was already proven in the past by Mathur et al. using AFM [37]. It is the opinion of the authors that assumption of elastic behaviour (within the limits of applicability) and use of Hertz model could be commonly accepted for comparative investigations, as in the case of the presented studies.

\subsection{Assessment of adhesive behaviour of cardiomyocytes}

From the first studies demonstrating AFM to have the sensitivity for investigating intermolecular forces [38], to the development of single cell probe force spectroscopy [39], biological samples have been widely characterized for their adhesive properties (see Fig. 2). In particular, the cell adhesion of CMs has been reported in some of the aforementioned studies [18,24,29]. Notably, in the study of Wu et al. [18], substrate coating did not affect cell adhesive properties

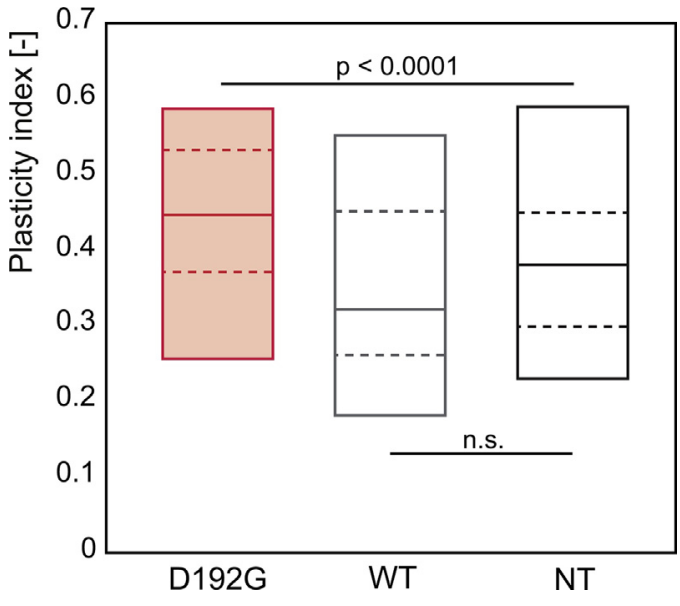

Fig. 3. Plasticity index for NRVMs expressing the human D192G lamin mutation compared to wild type (WT) and control (NT) cells. Data were acquired on $n>40$ cells for each condition. Statistical significance was probed with Kruskal-Wallis nonparametric test and Dunn's correction for multiple comparisons. A p-value $<0.05$ was assumed as statistically significant. n.s. = not significant.

in adult murine CMs. On the other hand, it was demonstrated that the geometry of the tip (sharp versus microbead) and the status of the cell (contracted versus relaxed) influence the adhesion of CMs, due to the different contact area between tip and sample and to the variation of active integrin receptors and costamere complexes during contractile cycle, respectively [18].

Vinculin was shown not to affect the initial peak adhesion force, while the probability of adhesion between AFM-fibronectin coated probe and a cell decreased significantly in vinculin null murine CMs compared to wild type specimens [24]. In the same study, the rescue of vinculin expression restored the adhesive properties to the level of wild type controls. Another protein, the human mutant LMNA, zeroed the adhesion work in NRVMs, whereas wild type and control cells exhibited similar values, as demonstrated by Lanzicher et al. [29].

\subsection{Assessment of plasticity index on cardiomyocytes}

The mechanical models used to estimate the elastic properties of a cell rely on strict assumptions, such as isotropy and homogeneity of the investigated sample, thus severely affecting the reliability of Young's modulus estimation. To overcome this limitation, Klymenko et al. [40] introduced the so-called index of plasticity, exploiting the hysteresis between loading and unloading force curves (Fig. 2). Such difference is attributed to an energy dissipation due to cytoskeletal organization. To the best of our knowledge, this parameter was measured by other groups in living cells [41,42], but not in cardiomyocytes. In particular, in the work of Mendez et al. [42], the plasticity index provided additional information to elucidate the role of vimentin in the viscoelastic response of mouse embryonic fibroblasts, thus demonstrating the relevance of this mechanical property.

We exploited the plasticity index to highlight the different behaviour of a living cell induced by a genetic mutation. The same protocol reported in $[29,43]$ was used to extract, infect and culture NRVMs expressing the human mutant D192G LMNA gene or the wild type form. Not infected (NT) NRVMs were used as further control. Measurements were performed directly on Petri dishes kept at physiological temperature. Cells were then indented on the nuclear region using a gold spherical probe (diameter around $5 \mu \mathrm{m}$ ) attached to a triangular cantilever (nominal spring constant $k=0.08 \mathrm{~N} / \mathrm{m}$ ). Data are displayed in Fig. 3 (original, unpublished 
data) and show that NRVMs expressing the mutant LMNA were significantly altered compared to controls.

\subsection{Viscoelasticity characterization of cardiomyocytes}

Besides the applications reported so far, the AFM has been used to characterise cells for their viscoelastic behaviour, indenting the sample and keeping either the height (stress relaxation) or the force (creep) constant for a fixed period of time [44] (see Fig. 2). Alternatively, after indentation, the cantilever can oscillate at small amplitude and variable frequency to describe the viscoelastic behaviour in terms of storage and loss moduli $[45,46]$. Despite this potentiality, to the best of our knowledge, only few examples of AFM rheological characterization of cardiomyocytes were reported in the literature.

In the work of Lanzicher, Martinelli et al. [43], stress relaxation experiments were performed on NRVMs infected with human LMNA D192G mutant gene. Relaxation data were fitted with the standard linear solid (SLS) model, also known as Zener's model. As compared to controls and cells expressing wild type human LMNA, mutant samples showed an increased purely elastic contribution, in accordance with Young's modulus values [29]. Moreover, cells with mutant A-type lamin exhibited a reduced viscosity, which was attributed to an impairment of length and thickness of the actin cytoskeleton elements induced by the lamin mutation.

The effect of different substrates (i.e. fibronectin- or collagencoated, aligned or not) and of ageing (from 1 to 15 days of culture) on the viscoelastic behaviour of neonatal rat CMs were assessed by Deitch et al. [47] through stress relaxation experiments. Here, the (normalized) relaxation data were fitted both with the Quasilinear Viscoelastic (QLV) and the SLS models to evaluate the percent relaxation after $60 \mathrm{~s}$ of load, the first resulting in a better fit of the experimental data. A decrease of the percent relaxation from day 1 to 3 in all samples was observed, then stabilizing to plateau values. CMs on aligned substrates displayed generally a lower relaxation than on random coating, since the actin fibres were more oriented, thus better counteracting the applied stress.

In a recent work by Robison et al. [48], AFM contributed to demonstrate the effect of detyrosination on buckling or sliding of microtubules during contraction in adult rat ventricular myocytes, where microtubules act as force bearing structures anchored to sarcomeric Z-disks by desmin. In particular, the SLS model was used to describe the elastic and viscous components as function of the indentation rate. Lowering the detyrosination level, both elasticity and viscosity of CMs were significantly reduced compared to controls. Depletion of desmin expression strongly reduced cell stiffness because of the missing connection between detyrosinated microtubules and sarcomeric Z-disks. On the other hand, on desmin-depleted CMs, viscosity did not vary after reduction of detyrosination levels.

Stress relaxation data can be used to predict the rheological behaviour of cells as function of frequency, assuming that the imposed deformation is within the linear regime. In the linear viscoelastic theory, the Boltzmann superposition is applicable and the relaxation modulus can be considered a predictive constituent parameter of the model for any arbitrary deformation history [49]. Relaxation data can be described using the Generalized Maxwell model, namely a purely elastic element (a spring) in parallel with Maxwell elements (a spring in series with a dashpot). Considering the integral form of the linear generalized viscoelastic model and an oscillating deformation of angular frequency $\omega$, the storage ( $E^{\prime}$ ) and the loss moduli (E") are calculated as:

$E^{\prime}(\omega)=E_{e}+\Sigma_{i=1}^{n} E_{i} \frac{\omega^{2} \tau_{i}^{2}}{1+\omega^{2} \tau_{i}^{2}}$

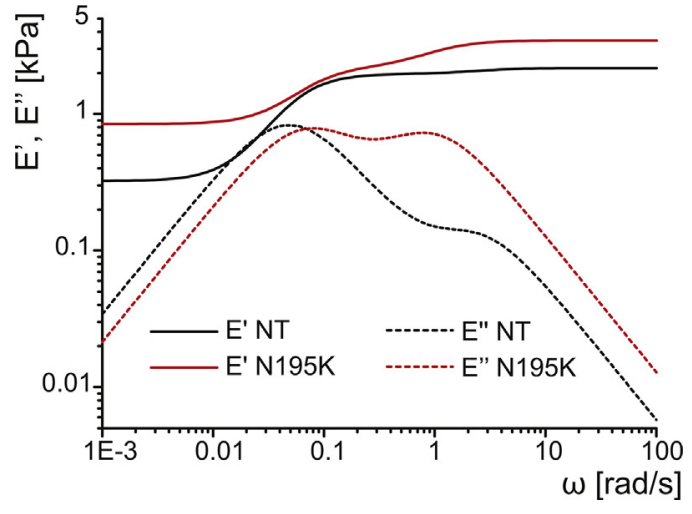

Fig. 4. Representative curves of storage (E', continuous lines) and loss (E", dashed lines) modulus profiles vs. the angular frequency $\omega$ for NRVMs bearing a mutation in the lamin gene (N195K, red curves) and in the native form (NT), used as control (black curves).

$E^{\prime \prime}(\omega)=\Sigma_{i=1}^{n} E_{i} \frac{\omega \tau_{i}}{1+\omega^{2} \tau_{i}^{2}}$

where $E_{e}$ is the relaxation modulus at equilibrium $(t \rightarrow \infty), n$ is the total number of Maxwell elements used to fit the relaxation profile, $E_{i}$ and $\tau_{i}$ are the elastic modulus and the relaxation time of the Maxwell element $i$. Thus, the elastic and viscous contributions of the mechanical response are easily separated and compared. As an example, we applied this model to predict the viscoelastic response of NRVMs after infection with human mutant N195K lamin, using the same protocol reported in $\S 3.3$. Results were compared to those obtained on not-treated samples (NT). Cells at physiological temperature were indented on the nuclear region with the spherical probe previously described, at $20 \%$ of their height. Relaxation force data were converted to moduli and fitted with a model consisting of one pure elastic element in parallel with two Maxwell elements, which demonstrated a better description of the initial part of the curve than a single Maxwell model. Resulting storage and loss moduli are reported in Fig. 4 (original, unpublished data), where a higher elastic contribution at both low and high frequency is displayed for mutant cells compared to controls. Even the viscous contribution for frequencies around $1 \mathrm{rad} / \mathrm{s}$ is more marked for mutant cells.

\subsection{AFM quantification of beating activity}

Monitoring the deflection of the cantilever positioned on a beating cardiomyocyte (or on a cluster of them) gives information about contractile force, frequency and vertical displacement of the cell. To the best of our knowledge, the first characterisation of beating profile was performed by Domke and co-authors [50], who measured the height change and the vertical deflection due to each pulse. Considering that contraction of adherent CMs is primarily a lateral displacement inducing a height change, Liu et al. [51] suggested the lateral (torsional) deflection of the cantilever as a more reliable signal to measure contractile activity. Nonetheless, most of the reported studies considered the vertical deflection, since the associated force can be easily calculated by multiplying the deflection signal by the sensitivity and the spring constant of the cantilever. Chang et al. [52] introduced the short-term Fourier transform to identify even small variations in the beating frequency, and suggested its application to detect CMs dynamics after drug administration.

The confluence level of CM culture is a key parameter affecting the beating profile: indeed, the frequency was shown to be much more regular on confluent cultures than on sub-confluent ones, whereas the effect on the beating amplitude seems controversial $[31,50,52]$. 
A deeper characterisation of the contractile behaviour of cardiomyocytes has been obtained coupling the AFM with other techniques. Very often, the atomic force microscopy is mounted on inverted microscopes, in order to perform simultaneous optical and mechanical investigation of the cells. The intracellular oscillation of $\mathrm{Ca}^{2+}$ ions can be monitored loading CMs with a proper calcium sensitive medium, as $\mathrm{Ca}^{2+}$ plays a fundamental role in contraction of the sarcomeric units that form the contractile apparatus of cardiomyocytes [53]. The aforementioned work of Liu and co-workers [51] demonstrated a linear relationship between the torsional force and the calcium flux intensity, whereas vertical deflection did not show such dependence. Burridge et al. [54] synchronized the mechanical and fluorescent signals, thus describing the time-dependence between calcium flux and force generation. AFM beating measurement was combined with digital holographic microscopy analysis by Yue et al. [22], overcoming the limits in resolution that affect the classical optical methods used to monitor contraction. In 2016, Tian and colleagues [55] coupled the AFM with a microelectrode array (MEA), thus proposing a novel and powerful method to simultaneously characterise the electrical and mechanical activity of contractile CMs. Although, some limitations were identified, particularly the presence of false peaks in the force and extracellular field potential profiles produced by cell clusters. These false peaks were absent when a single CM was deposited on the MEA.

AFM has been widely used to assess the role played by specific genes in the beating activity, as their mutations are often associated with cardiomyopathies. Nguyen et al. [56] showed that the knock-down of the stromal interaction molecule 1 (STIM1), a transmembrane protein on the sarcoplasmic reticulum acting as a $\mathrm{Ca}^{2+}$ sensor, decreased the beating regularity of HL-1 cardiac cells. The inhibition of the protein kinase $\mathrm{C} \varepsilon$ isoform (PKC $\varepsilon$ ), which is associated with a decreased phosphorylation of troponin $\mathrm{T}$ in ischaemic heart failure, was demonstrated to reduce the contraction amplitude in neonatal rat CMs [57]. Mutations in the cardiac troponin $\mathrm{T}$ can lead to dilated cardiomyopathies, thus Sun et al. [58] studied the effects of a point mutation of the TNNT2 gene, carried by a cohort of patients. Authors obtained cardiomyocytes from patient-specific iPSCs and studied them using AFM. DCM-derived CMs exhibited similar beating frequency and duration compared to healthy donors, but the contraction force was weaker. This was restored by inducing the overexpression of sarcoplasmic reticulum $\mathrm{Ca}^{2+}$ adenosine triphosphatase (Serca2a). Liu et al. [31] compared the beating profiles of single iPSC and ESC derived cardiomyocytes: despite the high variability, which resulted lower in CMs clusters, the two cell lines exhibited comparable beating force, while iPSC$\mathrm{CMs}$ demonstrated a slightly higher beat duration and a lower beat rate than ESC-CMs. A protocol to improve the homogeneity of embryoid bodies (EBs) to obtain beating cardiomyocytes both from human ESCs and iPSCs was proposed by Pesl et al. [59]. Even in this case, AFM measurements of the beating profile of EBs demonstrated comparable beating force in the two models, while the beat rate was higher for iPSCs derived CMs. Recently, the same group [60] proposed the use of EBs coupled to an AFM cantilever as a sensor to probe the effects of different environmental conditions (i.e. applied force, temperature, calcium level and drug treatments) on beating force and rate. Authors suggested this novel biosensor also for biomechanical characterisation of heart diseases and drug therapies, since EBs are closer to physiological conditions than single CMs on 2D cultures.

AFM studies were conducted to evaluate also viability and beating activity of CMs on supports purposely designed to deliver these cells to the injured cardiac tissue and repair it. Burridge et al. [54] used AFM to verify that interaction of iPSC-CMs with other cell types in a 3D hydrogel did not interfere with the contractile activity. Lin and co-workers [61] investigated the effect of size and orientation of electro-spun fibrous patches of gelatine-coated polyacrylonitrile on the beating frequency and force of neonatal rat myocytes. Authors found that CMs on aligned nanofibres beat faster and stronger than those on randomly oriented ones. The co-culture with endothelial cells also increased the frequency and force of the beating, whereas the use of micron-sized fibres strongly reduced the adhesion and the beating force, as nanofibres have dimensions closer to native cardiac extracellular matrix.

Detection of cardiomyocyte contractile activity can be also fruitfully used to evaluate the effects and/or cytotoxicity of drugs at the single cell level. Several studies demonstrated the reliability of AFM in recording the chronotropic (variation of frequency) and/or the inotropic (variation of beating force) actions of certain substances, suggesting its use for preclinical tests of candidate treatments. Studies reported in the literature used different CMs models, concentrations and administration approaches, so that a real quantification and comparison of the effects of each drug on the contraction force and the beat rate is cumbersome. As a general observation, drugs activating $\alpha 1$ and $\beta 1$ adrenergic receptors such as epinephrine (or norepinephrine, a demethylated form of epinephrine) led to an increase of both the beat rate and the contraction force $[31,52,55]$. The same effect was produced by isoproterenol $[55,57,59,60]$, a $\beta$ receptor agonist, which was shown to augment both the beat rate and contraction force after inhibition of Ser208 phosphorylation [57], or treatments with $\beta 1$ receptor blockers, such as metoprolol [59,60] or propranolol [55]. Moreover, caffeine enhanced the $\mathrm{Ca}^{2+}$ release from sarcomeric reticulum by activation of the RyR2 pathway, resulting in a fast increase of the contraction force, followed by a progressive decrease and leading to highly irregular beat frequency (arrhythmia) [59,60]. Other tested blocking agents were esmolol hydrochloride and doxazosin mesylate, acting on the $\beta$ and $\alpha$ adrenergic receptors, respectively, and leading to a decrease of both the beat rate and contraction amplitude [52]. Ibutilide, a drug used to treat arrhythmia, caused an increase of beating frequency and a reduction of the contraction force [51].

As previously stated, atomic force microscopy can be used to evaluate the toxicity of drugs on single CMs and to identify the best administration strategy to minimize the adverse effects. For instance, treatment with doxorubicin was demonstrated to significantly affect the beating properties of cardiomyocytes. Yue et al. [22] proved an increase of the beat duration and a quick intensification of the beating force in murine myocytes, followed by a strong decrease. Meanwhile, Chang et al. [52] found high irregularity and reduced contractile force in chicken embryonic myocytes. In the first case, the action of dexrazoxane as cardioprotective drug was proved, showing that its administration 30 min before doxorubicin treatment reduced the cardiotoxic effects. In the work of Chang and colleagues [52], the antioxidant action of vitamin C was shown to restore the contraction force of cardiomyocytes.

\section{Other applications of AFM on cardiomyocytes}

Beyond the already discussed assessment of contractile activity, AFM was employed to induce contraction in living cells, activating their mechano-sensitive ion channels. This was exploited by $\mathrm{He}$ et al. [62] to demonstrate the formation of membrane nanotubes as a transport mechanism between NRVMs and rat fibroblasts that are not strictly connected by gap junctions. Using simultaneous fluorescence imaging, authors showed that the $\mathrm{Ca}^{2+}$ signal from induced contraction propagated through the membrane nanotubes, thus leading the connected cells to contract. In 2015, Galie and co-workers [63] studied the effects of indentation depth and frequency for AFM induced contraction on 3D suspended constructs of NRVMs and rat fibroblasts. Authors found that indentation depth 
(from 1 to $3 \mu \mathrm{m}$ ) did not change either the contraction force or the contractile velocity, which was shown to increase with the indentation rate (from 0.5 to $2 \mathrm{~Hz}$ ).

Ossola and colleagues [64] exploited hollow cantilevers connected with a pressure control system (FluidFM technology) to develop an AFM-based patch-clamp device, that can characterise both electrophysiological and mechanical activity of contracting CMs. This setup took advantage from the force control loop of AFM for a more stable coupling between the hollow tip and the beating cell compared to the common patch clamp technique. The repeatability and the potential use of this technology as microinjector tool was demonstrated, while the limitations coming from the low electrical resistance in the cell-tip coupling were addressed for future improvements.

Recently, Smolyakov et al. [65] optimized the application of a novel multiparametric AFM mode for morphological and mechanical investigation of living and fixed adult murine cardiomyocytes. In the classical topological mapping with AFM, the force-volume method is exploited, where each pixel is probed by a forcedeformation curve. The alternative technique uses high frequency oscillating cantilevers for multiple probing on the same point. Such improvement allows higher resolution, reduced acquisition time and better contrast for cellular substructures in terms of height, stiffness, adhesion (related to the surface chemistry) and dissipation (related both to viscous and chemical effects) compared to the classical force-volume method. In the cited study, authors were able to clearly characterise even subsarcolemmal structures, such as mitochondria and sarcomeric apparatus, identifying both Z-lines and M-bands.

\section{Conclusion}

The interest in cardiovascular research is constantly fostered by the striking amount of deaths caused by cardiovascular diseases, therefore new methods and approaches are being pursued to better understand the pathophysiological conditions of the heart, both at the macro- and microscopic level.

The present work has been focused mainly on studies performed on single cells and cellular clusters, and reviewed most of the literature about cardiomyocytes investigation via AFM, ranging from the first works on CMs topography to the most recent ones exploiting advanced nanomechanical mapping. Reported data provided a thorough insight into cardiac physiological and pathological conditions, as well as the effects of therapeutic approaches at the cellular scale.

Nowadays, the topics hereby discussed are among the most pressing issues, and many open questions still require to be answered. Researchers in the field could take advantage of this review and the potential of AFM technique in order to test their hypotheses, achieve a deeper comprehension of cardiac behaviour and, hopefully, find new and effective therapies for cardiac pathologies.

\section{Conflict of interest statement}

The authors have no conflict of interest to declare.

\section{Fundings}

This work was supported by the "Fondation Leducq", Transatlantic Network of Excellence (grant number 14-CVD 03).

\section{Acknowledgements}

Authors gratefully thank Thomas Lanzicher for his contribution with the plasticity index data on NRVMs and Dr. Valentina Martinelli for providing the NRVMs used in this study.

\section{References}

[1] G. Binnig, C.F. Quate, C. Gerber, Atomic force microscope, Phys. Rev. Lett. 56 (1986) 930-933, http://dx.doi.org/10.1103/PhysRevLett.56.930.

[2] R. Benitez, J.L. Toca-herrera, Looking at cell mechanics with atomic force microscopy: experiment and theory, Microsc. Res. Tech. 77 (2014) 947-958, http://dx.doi.org/10.1002/jemt.22419.

[3] C.D. Gillespie, C. Wigington, Y. Hong, Coronary heart disease and stroke deaths-United States, 2009, CDC heal Disparities Inequalities Report-United States, vol. 62, 2013, pp. 157-160 https://www.cdc.gov/minorityhealth/ chdireport.html.

[4] D.P. Allison, N.P. Mortensen, C.J. Sullivan, M.J. Doktycz, Atomic force microscopy of biological samples, Wiley Interdiscip, Rev. Nanomed. Nanobiotechnol. 2 (2010) 618-634, http://dx.doi.org/10.1002/wnan.104.

[5] K.-C. Chang, Y.-W. Chiang, C.-H. Yang, J.-W. Liou, Atomic force microscopy in biology and biomedicine, Tzu Chi Med. J. 24 (2012) 162-169, http://dx.doi. org/10.1016/j.tcmj.2012.08.002.

[6] C. Perez-Terzic, A.M. Gacy, R. Bortolon, P.P. Dzeja, M. Puceat, M. Jaconi, F.G. Prendergast, A. Terzic, Structural plasticity of the cardiac nuclear pore complex in response to regulators of nuclear import, Circ. Res. 84 (1999) 1292-1301, http://dx.doi.org/10.1161/01.RES.84.11.1292.

[7] J.J. Davis, H.A.O. Hill, T. Powell, High resolution scanning force microscopy of cardiac myocytes, Cell Biol. Int. 25 (2001) 1271-1277, http://dx.doi.org/10. 1006/cbir.2001.0813.

[8] K. Kliche, M. Kuhn, U. Hillebrand, Y. Ludwig, C. Stock, H. Oberleithner, Direct aldosterone action on mouse cardiomyocytes detected with atomic force microscopy, Cell. Physiol. Biochem. 18 (2006) 265-274, http://dx.doi.org/10. $1159 / 000097673$.

[9] H. Yang, Y. Liu, X.L. Lu, X.H. Li, H.G. Zhang, Transmembrane transport of the G $\alpha$ q protein carboxyl terminus imitation polypeptide GCIP-27, Eur. J. Pharm. Sci. 49 (2013) 791-799, http://dx.doi.org/10.1016/j.ejps.2013.05.028.

[10] D.K. Lieu, J. Liu, C. Siu, G.P. McNerney, H. Tse, A. Abu-Khalil, T. Huser, R.A. Li, Absence of transverse tubules contributes to non-uniform $\mathrm{Ca}(2+)$ wavefronts in mouse and human embryonic stem cell-derived cardiomyocytes, Stem Cells Dev. 18 (2009) 1493-1500, http://dx.doi.org/10.1089/scd.2009.0052.

[11] E. Dague, G. Genet, V. Lachaize, C. Guilbeau-Frugier, J. Fauconnier, C. Mias, B. Payré, L. Chopinet, D. Alsteens, S. Kasas, C. Severac, J. Thireau, C. Heymes, B. Honton, A. Lacampagne, A. Pathak, J.M. Sénard, C. Galés, Atomic force and electron microscopic-based study of sarcolemmal surface of living cardiomyocytes unveils unexpected mitochondrial shift in heart failure, J. Mol. Cell. Cardiol. 74 (2014) 162-172, http://dx.doi.org/10.1016/j.yjmcc.2014. 05.006.

[12] L. Wang, T. Chen, X. Zhou, Q. Huang, C. Jin, Atomic force microscopy observation of lipopolysaccharide-induced cardiomyocyte cytoskeleton reorganization, Micron 51 (2013) 48-53, http://dx.doi.org/10.1016/j.micron. 2013.06.008.

[13] E.J. Wright, N.W. Hodson, M.J. Sherratt, M. Kassem, A.L. Lewis, C. Wallrapp, N. Malik, C.M. Holt, Combined MSC and GLP-1 therapy modulates collagen remodeling and apoptosis following myocardial infarction, Stem Cells Int. 2016 (2016) 1-12, http://dx.doi.org/10.1155/2016/7357096.

[14] N.A. Burnham, R.J. Colton, Measuring the nanomechanical properties and surface forces of materials using an atomic force microscope, J. Vac. Sci. Technol. A Vac. Surf. Film 7 (1989) 2906-2913, http://dx.doi.org/10.1116/1. 576168.

[15] G.R. Fedorchak, A. Kaminski, J. Lammerding, Cellular mechanosensing: getting to the nucleus of it all, Prog. Biophys. Mol. Biol. 115 (2014) 76-92, http://dx. doi.org/10.1016/j.pbiomolbio.2014.06.009.

[16] N. Gavara, A beginner's guide to atomic force microscopy probing for cell mechanics, Microsc. Res. Tech. (2016) 75-84, http://dx.doi.org/10.1002/jemt. 22776.

[17] S.G. Shroff, D.R. Saner, R. Lal, Dynamic micromechanical properties of cultured rat atrial myocytes measured by atomic force microscopy, Am. J. Physiol. Cell Physiol. 269 (1995) C286-292.

[18] X. Wu, Z. Sun, A. Foskett, J.P. Trzeciakowski, G.A. Meininger, M. Muthuchamy, Cardiomyocyte contractile status is associated with differences in fibronectin and integrin interactions, Am. J. Physiol. Heart Circ. Physiol. 298 (2010) H2071-H2081, http://dx.doi.org/10.1152/ajpheart.01156.2009.

[19] E.U. Azeloglu, K.D. Costa, Cross-bridge cycling gives rise to spatiotemporal heterogeneity of dynamic subcellular mechanics in cardiac myocytes probed with atomic force microscopy, Am. J. Physiol. Heart Circ. Physiol. 298 (2010) H853-H860, http://dx.doi.org/10.1152/ajpheart.00427.2009.

[20] J.F. Saenz Cogollo, M. Tedesco, S. Martinoia, R. Raiteri, A new integrated system combining atomic force microscopy and micro-electrode array for measuring the mechanical properties of living cardiac myocytes, Biomed. Microdevices 13 (2011) 613-621, http://dx.doi.org/10.1007/s10544-011-9531-9. 
[21] U.G. Hofmann, C. Rotsch, W.J. Parak, M. Radmacher, Investigating the cytoskeleton of chicken cardiocytes with the atomic force microscope, J. Struct. Biol. 119 (1997) 84-91, http://dx.doi.org/10.1006/jsbi.1997.3868.

[22] T. Yue, K.H. Park, B.E. Reese, H. Zhu, S. Lyon, J. Ma, P.J. Mohler, M. Zhang Quantifying drug-induced nanomechanics and mechanical effects to single cardiomyocytes for optimal drug administration to minimize cardiotoxicity, Langmuir 32 (2016) 1909-1919, http://dx.doi.org/10.1021/acs.langmuir. 5 b04314.

[23] X. Shi, L. Qin, X. Zhang, K. He, C. Xiong, J. Fang, X. Fang, Y. Zhang, Elasticity of cardiac cells on the polymer substrates with different stiffness: an atomic force microscopy study, Phys. Chem. Chem. Phys. 13 (2011) 7540-7545, http://dx.doi.org/10.1039/c1cp20154a.

[24] J.R. Tangney, J.S. Chuang, M.S. Janssen, A. Krishnamurthy, P. Liao, M. Hoshijima, X. Wu, G.A. Meininger, M. Muthuchamy, A. Zemljic-Harpf, R.S. Ross, L.R. Frank, A.D. McCulloch, J.H. Omens, Novel role for vinculin in ventricular myocyte mechanics and dysfunction, Biophys. J. 104 (2013) 1623-1633, http://dx.doi.org/10.1016/j.bpj.2013.02.021.

[25] G. Genet, C. Guilbeau-Frugier, B. Honton, E. Dague, M.D. Schneider, C. Coatrieux, D. Calise, C. Cardin, C. Nieto, B. Payré, C. Dubroca, P. Marck, C. Heymes, A. Dubrac, D. Arvanitis, F. Despas, M.F. Altié, M.H. Seguelas, M.B. Delisle, A. Davy, J.M. Sénard, A. Pathak, C. Galés, Ephrin-B1 is a novel specific component of the lateral membrane of the cardiomyocyte and is essential for the stability of cardiac tissue architecture cohesion, Circ. Res. 110 (2012) 688-700, http://dx.doi.org/10.1161/CIRCRESAHA.111.262451.

[26] S.C. Lieber, N. Aubry, J. Pain, G. Diaz, S.-J. Kim, S.F. Vatner, Aging increases stiffness of cardiac myocytes measured by atomic force microscopy nanoindentation, Am. J. Physiol. Heart Circ. Physiol. 287 (2004) H645-H651, http://dx.doi.org/10.1152/ajpheart.00564.2003.

[27] M.E. Nance, J.T. Whitfield, Y. Zhu, A.K. Gibson, L.M. Hanft, K.S. Campbell, G.A. Meininger, K.S. McDonald, S.S. Segal, T.L. Domeier, Attenuated sarcomere lengthening of the aged murine left ventricle observed using two-photon fluorescence microscopy, Am. J. Physiol. Heart Circ. Physiol. 309 (2015) H918-H925, http://dx.doi.org/10.1152/ajpheart.00315.2015.

[28] J.C. Benech, N. Benech, A.I. Zambrana, I. Rauschert, V. Bervejillo, N. Oddone, J.P. Damian, Diabetes increases stiffness of live cardiomyocytes measured by atomic force microscopy nanoindentation, AJP Cell Physiol. 307 (2014) C910-C919, http://dx.doi.org/10.1152/ajpcell.00192.2013.

[29] T. Lanzicher, V. Martinelli, L. Puzzi, G. Del Favero, B. Codan, C.S. Long, L. Mestroni, M.R.G. Taylor, O. Sbaizero, The cardiomyopathy lamin A/C D192G mutation disrupts whole-cell biomechanics in cardiomyocytes as measured by atomic force microscopy loading-unloading curve analysis, Sci. Rep. 5 (2015) 13388, http://dx.doi.org/10.1038/srep13388.

[30] W. Sumita Yoshikawa, K. Nakamura, D. Miura, J. Shimizu, K. Hashimoto, N. Kataoka, H. Toyota, H. Okuyama, T. Miyoshi, H. Morita, K. Fukushima Kusano, T. Matsuo, M. Takaki, F. Kajiya, N. Yagi, T. Ohe, H. Ito, Increased passive stiffness of cardiomyocytes in the transverse direction and residual actin and myosin cross-bridge formation in hypertrophied rat hearts induced by chronic $\beta$-adrenergic stimulation, Circ. J. 77 (2013) 741-748, http://dx.doi. org/10.1253/circj.CJ-12-0779.

[31] J. Liu, N. Sun, M.A. Bruce, J.C. Wu, M.J. Butte, Atomic force mechanobiology of pluripotent stem cell-derived cardiomyocytes, PLoS One 7 (2012) 1-7, http:// dx.doi.org/10.1371/journal.pone.0037559.

[32] I.V. Ogneva, T.M. Mirzoev, N.S. Biryukov, O.M. Veselova, I.M. Larina, Structure and functional characteristics of rat's left ventricle cardiomyocytes under antiorthostatic suspension of various duration and subsequent reloading, $\mathrm{J}$. Biomed. Biotechnol. 2012 (2012) 1-11, http://dx.doi.org/10.1155/2012/ 659869.

[33] I.V. Ogneva, N.S. Biryukov, T.A. Leinsoo, I.M. Larina, Possible role of non-muscle alpha-actinins in muscle cell mechanosensitivity, PLoS One 9 (2014), http://dx.doi.org/10.1371/journal.pone.0096395.

[34] I.V. Ogneva, V. Gnyubkin, N. Laroche, M.V. Maximova, I.M. Larina, L. Vico, Structure of the cortical cytoskeleton in fibers of postural muscles and cardiomyocytes of mice after 30-day 2-g centrifugation, J. Appl. Physiol. 118 (2015) 613-623, http://dx.doi.org/10.1152/japplphysiol.00812.2014.

[35] V. Szuts, F. Ötvös, O. Bencsik, G. Váró, J.A. Jarabin, A. Kovács, L. Rovó, J.G. Kiss, T. Szénási, A. Véha, A. Szekeres, C. Vágvölgy, K. Halasy, Z. Szegletes, Effects of 6-epi-ophiobolin A on mechanical and physiological parameters of cardiomyocytes and on the reorganization and amounts of their kv4.x ion channels, Acta Biol. Szeged. 60 (2016) 157-166.

[36] A.A. Soufivand, M. Navidbakhsh, M. Soleimani, Is it appropriate to apply Hertz model to describe cardiac myocytes' mechanical properties by atomic force microscopy nanoindentation? Micro Nano Lett. 9 (2014) 153-156, http://dx. doi.org/10.1049/mnl.2014.0019.

[37] A.B. Mathur, A.M. Collinsworth, W.M. Reichert, W.E. Kraus, G.A. Truskey, Endothelial, cardiac muscle and skeletal muscle exhibit different viscous and elastic properties as determined by atomic force microscopy, J. Biomech. 34 (2001) 1545-1553, http://dx.doi.org/10.1016/S0021-9290(01)00149-X.

[38] J.H. Hoh, J.P. Cleveland, C.B. Prater, J.P. Revel, P.K. Hansma, Quantized adhesion detected with the atomic force microscope, J. Am. Chem. Soc. 114 (1992) 4917-4918, http://dx.doi.org/10.1021/ja00038a075.

[39] W.R. Bowen, N. Hilal, R.W. Lovitt, C.J. Wright, Direct measurement of the force of adhesion of a single biological cell using an atomic force microscope Colloids Surf. A Physicochem. Eng. Asp. 136 (1998) 231-234, http://dx.doi. org/10.1016/S0927-7757(97)00243-4.
[40] O. Klymenko, J. Wiltowska-Zuber, M. Lekka, W.M. Kwiatek, Energy dissipation in the AFM elasticity measurements, Acta Phys. Pol. A 115 (2009) 548-551, http://dx.doi.org/10.12693/APhysPolA.115.548.

[41] A.N. Fernandes, X. Chen, C.A. Scotchford, J. Walker, D.M. Wells, C.J. Roberts, N.M. Everitt, Mechanical properties of epidermal cells of whole living roots of Arabidopsis thaliana: an atomic force microscopy study, Phys. Rev. E 85 (2012) 21916, http://dx.doi.org/10.1103/PhysRevE.85.021916.

[42] M.G. Mendez, D. Restle, P.A. Janmey, Vimentin enhances cell elastic behavior and protects against compressive stress, Biophys. J. 107 (2014) 314-323, http://dx.doi.org/10.1016/j.bpj.2014.04.050.

[43] T. Lanzicher, V. Martinelli, C.S. Long, G. Del Favero, L. Puzzi, M. Borelli, L Mestroni, M.R.G. Taylor, O. Sbaizero, AFM single-cell force spectroscopy links altered nuclear and cytoskeletal mechanics to defective cell adhesion in cardiac myocytes with a nuclear lamin mutation, Nucleus 6 (2015) 394-407, http://dx.doi.org/10.1080/19491034.2015.1084453.

[44] S. Moreno-Flores, R. Benitez, M. dM Vivanco, J.L. Toca-Herrera, Stress relaxation and creep on living cells with the atomic force microscope: a means to calculate elastic moduli and viscosities of cell components, Nanotechnology 21 (2010) 445101, http://dx.doi.org/10.1088/0957-4484/21/ $44 / 445101$

[45] J. Alcaraz, L. Buscemi, M. Grabulosa, X. Trepat, B. Fabry, R. Farré, D. Navajas, Microrheology of human lung epithelial cells measured by atomic force microscopy, Biophys. J. 84 (2003) 2071-2079, http://dx.doi.org/10.1016/ S0006-3495(03)75014-0.

[46] B.A. Smith, B. Tolloczko, J.G. Martin, P. Grütter, Probing the viscoelastic behavior of cultured airway smooth muscle cells with atomic force microscopy: stiffening induced by contractile agonist, Biophys. J. 88 (2005) 2994-3007, http://dx.doi.org/10.1529/biophysj.104.046649.

[47] S. Deitch, B.Z. Gao, D. Dean, Effect of matrix on cardiomyocyte viscoelastic properties in 2D culture, Mol. Cell. Biomech. 9 (2012) 227-249.

[48] P. Robison, M.A. Caporizzo, H. Ahmadzadeh, A.I. Bogush, C.Y. Chen, K.B. Margulies, V.B. Shenoy, B.L. Prosser, Detyrosinated microtubules buckle and bear load in contracting cardiomyocytes, Science 352 (2016) aaf0659, http:// dx.doi.org/10.1126/science.aaf0659.

[49] R.B. Bird, R.C. Armstrong, O. Hassager, Fluid mechanics Dynamics of Polymeric Liquids, vol. 1, 2nd ed., Wiley-Interscience, New York, 1987 (Chapter 6).

[50] J. Domke, W.J. Parak, M. George, H.E. Gaub, M. Radmacher, Mapping the mechanical pulse of single cardiomyocytes with the atomic force microscope, Eur. Biophys. J. 28 (1999) 179-186, http://dx.doi.org/10.1007/s002490050198.

[51] Y. Liu, J. Feng, L. Shi, R. Niu, Q. Sun, H. Liu, J. Li, J. Guo, J. Zhu, D. Han, In situ mechanical analysis of cardiomyocytes at nano scales, Nanoscale 4 (2012) 99-102, http://dx.doi.org/10.1039/C1NR11303H.

[52] W.-T. Chang, D. Yu, Y.-C. Lai, K.-Y. Lin, I. Liau, Characterization of the mechanodynamic response of cardiomyocytes with atomic force microscopy, Anal. Chem. 85 (2013) 1395-1400, http://dx.doi.org/10.1021/ac3022532.

[53] N. Frey, T. a McKinsey, E.N. Olson, Decoding calcium signals involved in cardiac growth and function, Nat. Med. 6 (2000) 1221-1227, http://dx.doi. org/10.1038/81321.

[54] P.W. Burridge, S.A. Metzler, K.H. Nakayama, O.J. Abilez, C.S. Simmons, M.A Bruce, Y. Matsuura, P. Kim, J.C. Wu, M. Butte, N.F. Huang, P.C. Yang, Multi-cellular interactions sustain long-term contractility of human pluripotent stem cell-derived cardiomyocytes, Am. J. Transl. Res. 6 (2014) 724-735, http://dx doi.org/10.1016/j.tem.2013.11.001.

[55] J. Tian, C. Tu, B. Huang, Y. Liang, J. Zhou, X. Ye, Study of the union method of microelectrode array and AFM for the recording of electromechanical activities in living cardiomyocytes, Eur. Biophys. J. (2016), http://dx.doi.org/ 10.1007/s00249-016-1192-4.

[56] N. Nguyen, M. Biet, É. Simard, É. Béliveau, N. Francoeur, G. Guillemette, R. Dumaine, M. Grandbois, G. Boulay, STIM1 participates in the contractile rhythmicity of HL-1 cells by moderating T-type Ca2+ channel activity, Biochim. Biophys. Acta - Mol. Cell Res. 2013 (1833) 1294-1303, http://dx.doi. org/10.1016/j.bbamcr.2013.02.027.

[57] E. Dubois-Deruy, A. Belliard, P. Mulder, M. Bouvet, C. Smet-Nocca, S. Janel, F. Lafont, O. Beseme, P. Amouyel, V. Richard, F. Pinet, Interplay between troponin $\mathrm{T}$ phosphorylation and $\mathrm{O}-\mathrm{N}$-acetylglucosaminylation in ischaemic heart failure, Cardiovasc. Res. 107 (2015) 56-65, http://dx.doi.org/10.1093/ cvr/cvv136.

[58] N. Sun, M. Yazawa, J. Liu, L. Han, V. Sanchez-Freire, O.J. Abilez, E.G. Navarrete, S. Hu, L. Wang, A. Lee, A. Pavlovic, S. Lin, R. Chen, R.J. Hajjar, M.P. Snyder, R.E. Dolmetsch, M.J. Butte, E.A. Ashley, M.T. Longaker, R.C. Robbins, J.C. Wu, Patient-specific induced pluripotent stem cells as a model for familial dilated cardiomyopathy, Sci. Transl. Med. 4 (2012), http://dx.doi.org/10.1126/ scitranslmed.3003552 (130ra47-130ra47).

[59] M. Pesl, I. Acimovic, J. Pribyl, R. Hezova, A. Vilotic, J. Fauconnier, J. Vrbsky, P. Kruzliak, P. Skladal, T. Kara, V. Rotrekl, A. Lacampagne, P. Dvorak, A.C. Meli, Forced aggregation and defined factors allow highly uniform-sized embryoid bodies and functional cardiomyocytes from human embryonic and induced pluripotent stem cells, Heart Vessels 29 (2013) 834-846, http://dx.doi.org/10. 1007/s00380-013-0436-9.

[60] M. Pesl, J. Pribyl, I. Acimovic, A. Vilotic, S. Jelinkova, A. Salykin, A. Lacampagne, P. Dvorak, A.C. Meli, P. Skladal, V. Rotrekl, Atomic force microscopy combined with human pluripotent stem cell derived cardiomyocytes for biomechanical sensing, Biosens. Bioelectron. 85 (2016) 751-757, http://dx.doi.org/10.1016/j. bios.2016.05.073

[61] Y. Lin, M. Ko, S.-T. Wu, S. Li, J. Hu, Y. Lai, H.I. Harn, I. Laio, M.-L. Yeh, H. Yeh, M. Tang, K. Chang, F. Su, E.I.H. Wei, S.-T. Lee, J. Chen, A.S. Hoffman, W. Wu, P.C.H. 
Hsieh, A nanopatterned cell-seeded cardiac patch prevents electro-uncoupling and improves the therapeutic efficacy of cardiac repair, Biomater. Sci. 2 (2014) 567-580, http://dx.doi.org/10.1039/c3bm60289c.

[62] K. He, X. Shi, X. Zhang, S. Dang, X. Ma, F. Liu, M. Xu, Z. Lv, D. Han, X. Fang, Y. Zhang, Long-distance intercellular connectivity between cardiomyocytes and cardiofibroblasts mediated by membrane nanotubes, Cardiovasc. Res. 92 (2011) 39-47, http://dx.doi.org/10.1093/cvr/cvr189.

[63] P.A. Galie, F.J. Byfield, C.S. Chen, J.Y. Kresh, P.A. Janmey, Mechanically stimulated contraction of engineered cardiac constructs using a microcantilever, IEEE Trans. Biomed. Eng. 62 (2015) 438-442, http://dx.doi. org/10.1109/tbme.2014.2357778.
[64] D. Ossola, M.Y. Amarouch, P. Behr, J. Vörös, H. Abriel, T. Zambelli, Force-controlled patch clamp of beating cardiac cells, Nano Lett. 15 (2015) 1743-1750, http://dx.doi.org/10.1021/nl504438z.

[65] G. Smolyakov, M. Cauquil, C. Severac, V. Lachaize, C. Guilbeau-Frugier, J. Sénard, C. Galés, E. Dague, Biophysical properties of cardiomyocyte surface explored by multiparametric AFM, J. Struct. Biol. 198 (2017) 28-37, http://dx doi.org/10.1016/j.jsb.2017.03.001. 